\title{
Portal Hypertension and Chronic Kidney Disease Significantly Increase the Risk of Early Unplanned Readmissions in GAVE- Related Admissions
}

\author{
Lauren Pioppo ${ }^{1}$, Abhishek Bhurwal ${ }^{2}$, Hemant Raj Mutneja ${ }^{3}$, Puru Rattan ${ }^{2}$, Debashis Reja ${ }^{1}$, Augustine Tawadros ${ }^{1}$, Anish \\ Patel $^{2}$, Vinod Rustgi ${ }^{2}$
}

1) Department of Internal

Medicine, Rutgers Robert

Wood Johnson Medical

School, New Brunswick, NJ

2) Department of

Gastroenterology and

Hepatology, Rutgers Robert

Wood Johnson Medical

School, New Brunswick, NJ

3) Department of

Gastroenterology and

Hepatology, John H Stroger

Cook County Hospital,

Chicago, IL, USA

\author{
Address for correspondence: \\ Vinod Rustgi, MD, MBA \\ Department of \\ Gastroenterology and \\ Hepatology, \\ Rutgers Robert Wood Johnson \\ Medical School, \\ New Brunswick, NJ \\ vr262@rwjms.rutgers.edu
}

Received: 03.02.2020

Accepted: 20.04.2020

\begin{abstract}
Background \& Aims: Gastric antral vascular ectasia (GAVE) is an uncommon cause of non-variceal upper gastrointestinal bleeding that is characterized by dilation of blood vessels in the antrum of the stomach. Various co-morbidities are associated with the development of GAVE, but the impact of co-morbidities on unplanned GAVE readmissions is unclear. The aim of this study was to assess the national incidence, 30-day mortality rate, and 30-day readmissions related to GAVE. Secondary outcomes were evaluation of predictors of early readmission, hospital length of stay (LOS) and total hospitalization charges.

Methods: Using the 2016 National Readmission Database, we analyzed discharges for GAVE. ICD-10 CM codes were utilized to identify associated comorbidities and inpatient procedures during the index admission. 30-day readmissions were identified for GAVE. Secondary measures of outcomes including LOS and hospitalization charges were also calculated. Risk factors for early readmission were also evaluated using multivariate analysis to adjust for confounders.

Results: A total of 18,375 index admissions for GAVE were identified. 20.49\% ( $n=3,720)$ of the discharged patients were readmitted within 30 days. 30-day mortality of GAVE-related admissions was $1.82 \%(n=335)$. Early readmissions accounted for 20,157 hospital days along with $\$ 189$ million in hospitalization costs. Multivariate analysis revealed that the presence of portal hypertension (OR 1.63; 95\% CI 1.37-1.93; $\mathrm{p}=0.0001$ ) and chronic kidney disease (CKD) (OR 1.62, 95\% CI 1.44-1.82; $\mathrm{p}<0.0001)$ significantly increased the odds of early readmission.

Conclusions: Our analysis demonstrates that the overall 30-day mortality rate of GAVE-related admissions is relatively low, but the 30-day readmission rate is significantly high. Patients with comorbid CKD and portal hypertension have a significantly higher risk of readmission. Further studies are required to determine if therapeutic interventions such as argon plasma coagulation or radiofrequency ablation during the index admission may prevent readmissions in these specific subgroups.
\end{abstract}

Key words: gastric antral vascular ectasia - portal hypertension - renal insufficiency - chronic, hemorrhage - autoimmune diseases - mortality - incidence - endoscopy.

Abbreviations: AHRQ: Agency of Healthcare Research and Quality; ANA: antinuclear antibody; APC: argon plasma coagulation; CAD: coronary artery disease; CI: confidence interval; CKD: chronic kidney disease; GAVE: gastric antral vascular ectasia; HCUP: Healthcare Cost and Utilization Project; ICD-10-CM: International Classification of diseases, Tenth Revision, Clinical Modification; LOS: length of stay; MGUS: monoclonal gammopathy of undetermined significance; NAFLD: nonalcoholic fatty liver disease; NRD: National Readmission Database; OR: odds ratio; PBC: primary biliary cholangitis; PHG: portal hypertensive gastropathy; RA: rheumatoid arthritis; RFA: radiofrequency ablation; SLE: systemic lupus erythematous; UGIB: upper gastrointestinal bleeding.

\section{INTRODUCTION}

Gastric antral vascular ectasia (GAVE) is a microvascular deformity of the gastric mucosa [1]. While the exact cause and pathogenesis of GAVE are poorly understood, it is characterized by dilatation of the mucosal capillaries and tortuous submucosal veins mainly in the gastric antrum [2, 3]. GAVE is usually diagnosed endoscopically and is classically delineated into two main phenotypes: linear and punctate [4]. Due to the alternating stripes of red and pink seen in the linear type, it has 
been nicknamed "watermelon stomach," whereas the punctate phenotype appears as petechial-like lesions in the antrum [1]. Histologic findings reveal fibromuscular proliferation in the lamina propria, ectatic and dilated capillaries and fibrin microthrombi [1,5-9]. More recently, a third phenotype has been described, which appears as benign-appearing antral mucosal nodules that are virtually endoscopically indistinguishable from other benign nodules [10].

GAVE has a broad range of clinical presentations from asymptomatic occult blood loss to overt upper gastrointestinal bleeding (UGIB). However, GAVE is a relatively uncommon cause of gastrointestinal bleeding representing about $4 \%$ of all UGIB [11]. Often, this condition is incidentally diagnosed during endoscopy for the work-up for occult gastrointestinal bleeding or unexplained iron-deficiency anemia.

Prior studies have shown that GAVE has a female predominance and most commonly occurs at the age of 80 years [11-14]. The most common comorbidities associated with GAVE are cirrhosis, autoimmune diseases, vascular diseases, kidney disease, and diabetes mellitus [13-18]. GAVE is also associated with portal hypertension regardless of the presence of cirrhosis and is observed in patients with chronic liver disease without portal hypertension [6]. While the clinical manifestations are similar, GAVE has been described as a distinct entity from portal hypertensive gastropathy (PHG) which typically affects the fundus but can also be found in other areas of the gastrointestinal tract [19-23]. PHG is more frequently encountered than GAVE, and as its name implies, is only seen in the setting of portal hypertension [24,25].

Management of GAVE is clinically challenging. Treatment options for GAVE include medical, surgical and most commonly, endoscopic options. Surgery typically consists of antrectomy which is associated with high morbidity and mortality and is thus reserved for severe, refractory cases. There is little evidence for effectiveness of medical therapy, such as estrogen and progesterone therapy, in GAVE. Thus, the main treatment for GAVE remains endoscopic therapy such as argon plasma coagulation (APC) or radiofrequency ablation (RFA) [3, 26].

We aimed to analyze the 30-day readmission rate and 30day mortality rate after an episode of GAVE-related bleeding as well as the most common reasons for early unplanned readmission using the National Readmission Database (NRD). This is the largest publicly available readmission database in the United States. It is part of the Healthcare Cost and Utilization Project (HCUP) which is sponsored by the Agency of Healthcare Research and Quality (AHRQ). National Readmission Database can be used to generate national estimates of readmissions, reasons for returning to the hospital and hospital costs for discharges [27]. Furthermore, we analyzed the factors associated with early readmissions and the impact on health care utilization of hospitalization charges.

\section{METHODS}

\section{Data Source}

We analyzed the 2016 NRD which included 35,660,906 discharges in the United States [27]. The database is generated from the HCUP State Inpatient Databases which contain verified patient linkage numbers which can be used to track the patient across the hospitals within the state [28]. These discharges contain information regarding demographics, principal and secondary diagnoses, procedures, length of stay, in-patient mortality, readmissions and hospital costs. Each discharge is then weighted to represent the national sample. The diagnoses and procedure codes are collected using the International Classification of diseases, Tenth Revision, Clinical Modification (ICD-10-CM). All the discharges are put together in a stratified probability sample to represent all nonfederal acute care hospitalizations across the country.

\section{Study population}

Patients over the age of 18 with a principal discharge diagnosis of GAVE (K31.819) were included. All patients less than 18 years of age and elective admissions were excluded in the study. The NRD captures all the admissions for the calendar year 2016 (January 1 through December 31) and does not have linkage to the prior or next year. Therefore, index hospitalization discharges in the month of December were excluded.

The NRD variables were used to identify patient's age (in years), gender, median household income, primary expected payer, area of residence, hospital size and teaching status. Length of stay and total hospitalization charges are coded in the NRD [27]. ICD-10-CM codes were used to identify comorbidities and the procedures that were performed during the admissions. These data are shown in Supplement Table I.

A readmission was defined as any non-traumatic admission for any principal diagnosis within 30 days of the discharge. In the scenario where the patient had multiple admissions within 30 days of discharge, only the first readmission was considered. The denominator for the readmission rate excluded all patients who had died during the index admission. The 30-day mortality rate was calculated by utilizing the patient status within 30 days of the index admission. The principal diagnoses of all readmissions were tallied to calculate the 5 most common reasons for readmission. Rebleeding was defined as readmission with principal diagnosis of GAVE.

\section{Study Outcomes}

The primary outcome was all-cause 30-day unplanned early readmission after index hospitalization for GAVE-related bleeding. The secondary outcomes were: (1) in-hospital mortality during index admission and readmission, (2) 30day mortality rate, (3) the 5 most common principal causes of readmission, (4) resource utilization with readmissions and (5) independent risk factors for readmission.

\section{Statistics}

All statistics were calculated using STATA version 15.0 (StataCorp, College Station, TX). The NRD is a complex weighted sampling design that has stratification and clustering. This yields a nationally representative analysis of unbiased results, variance and p- values. These weighted observations were used to calculate all estimates for the hospitalized patients with GAVE-related bleeding. Continuous variables were evaluated using student-t test whereas categorical variables were evaluated using chi-square test. Subsequently, 
multivariable regression analysis was used to adjust for confounders. All p-values were 2-sided with 0.05 as the threshold for statistical significance.

\section{RESULTS}

A total of 35,660,906 admissions were screened, and 18,375 index admissions for GAVE were identified and included in the study. ICD-10 CM codes used to screen patients are outlined in Supplement Table I. The 30-day mortality of GAVE-related admissions was $1.82 \%(n=335)$. A total of $20.49 \%(n=3,720)$ of the discharged patients were readmitted within 30 days.

The most common cause of readmission within 30 days was GAVE-related rebleeding (16.3\%). Other causes were unspecified hemorrhage (6.3\%), sepsis (4.1\%), melena (3.3\%), angiodysplasia of the colon $(3.3 \%)$.

Patient demographics including age, gender and comorbidities of the index and readmissions are outlined in Table I. There was no significant difference in gender between the index and readmission group $(\mathrm{p}=0.539)$. Patients that were readmitted tended to be slightly younger on average, about 71 years compared to 72 years (OR 0.98 95\%, CI 0.98-0.99, $\mathrm{p}=0.0001)$.

Early readmissions accounted for 20,157 hospital days along with $\$ 189$ million in hospitalization costs. The mean length of stay for the index cohort was 5.21 days as compared to 5.41 days for the readmitted cohort. The mean hospitalization cost for the index cohort was $\$ 50,988$ as compared to $\$ 51,554$ for the unplanned readmitted group.
On univariate analysis, readmitted patients had a higher prevalence of portal hypertension, systemic lupus erythematous (SLE) and rheumatoid arthritis (RA). These patients also had lower prevalence of Sjogren's syndrome, Raynaud's syndrome, polymyalgia rheumatica, and CREST (calcinosis, Raynaud's phenomenon, esophageal dysmotility, sclerodactyly, and telangiectasia). Despite the adjustment of confounders, patients who had portal hypertension or chronic kidney disease (CKD) had a higher risk of 30-day readmission (OR 1.63; 95\% CI 1.37-1.93; p=0.0001, OR 1.62, 95\% CI 1.44$1.82 ; \mathrm{p}<0.0001$, respectively). However, subgroup analysis did not reveal any specific etiology of portal hypertension that leads to increased readmission rate including nonalcoholic fatty liver disease (NAFLD), autoimmune hepatitis, or alcoholic cirrhosis (Table II). There was no statistically significant increase in the risk of early readmissions in patients with GAVE and typical comorbid conditions such as systemic sclerosis, Raynaud's syndrome, SLE, primary biliary cholangitis (PBC), polymyalgia rheumatica, monoclonal gammopathy of undetermined significance (MGUS), RA, or history of bone marrow transplant (Table II). Endoscopy during the index admission performed for GAVE did not decrease odds of 30day readmission.

A total of $1.21 \%(n=223)$ patients died during the index admission for GAVE related admissions. 30-day mortality for GAVE was $1.82 \%(n=335)$. The mortality rate during readmissions was $3.7 \%(\mathrm{n}=138)$.

Table III shows the results of the multivariate regression analysis of factors associated with mortality in index

Table I. Demographic differences between index cases and readmissions

\begin{tabular}{|c|c|c|c|}
\hline Characteristics & $\begin{array}{l}\text { Index Admissions } \\
\text { without readmissions }\end{array}$ & $\begin{array}{l}\text { Index admissions } \\
\text { with readmissions }\end{array}$ & $\mathrm{p}$ \\
\hline Females (\%) & 51.17 & 50.34 & 0.88 \\
\hline Mean age (years) & 72.73 & 71.47 & 0.001 \\
\hline Portal hypertension (\%) & 0.98 & 12.1 & 0.0001 \\
\hline Systemic sclerosis (\%) & 0.45 & 0.49 & 0.57 \\
\hline Sjogren syndrome (\%) & 0.24 & $<0.01$ & 0.0009 \\
\hline Raynaud's syndrome (\%) & 0.56 & 0.3 & 0.0002 \\
\hline Systemic lupus erythematous (\%) & 0.5 & 0.62 & 0.0087 \\
\hline Primary biliary cholangitis (\%) & 0.43 & 0.54 & 0.12 \\
\hline History of bone marrow transplant (\%) & 0.13 & 0.19 & 0.14 \\
\hline Coronary artery disease (\%) & 41.27 & 40.05 & 0.88 \\
\hline Polymyalgia rheumatica (\%) & 0.68 & 0.42 & 0.0003 \\
\hline $\begin{array}{l}\text { Monoclonal gammopathy of undetermined } \\
\text { significance }(\%)\end{array}$ & 0.54 & 0.5 & 0.57 \\
\hline Hyperlipidemia (\%) & 44.11 & 39.17 & 0.47 \\
\hline Chronic kidney disease (\%) & 41.3 & 49.04 & 0.87 \\
\hline Rheumatoid arthritis (\%) & 0.68 & 2.13 & $<0.0001$ \\
\hline CREST (\%) & 0.29 & $<0.01$ & 0.0008 \\
\hline Diabetes mellitus & 40.68 & 44.65 & 0.89 \\
\hline Peripheral vascular disease & 10.85 & 9.99 & 0.87 \\
\hline
\end{tabular}

Index cases include those admitted for GAVE-related bleeding that were not readmitted. This is compared to index cases admitted for GAVE-related bleeding that were readmitted within 30 days; GAVE: gastric antral vascular ectasia; CREST: calcinosis, Raynaud's phenomenon, esophageal dysmotility, sclerodactyly, and telangiectasia. 
admissions with GAVE. The presence of autoimmune hepatitis was associated with significantly increased odds of mortality in patients with GAVE (OR 9.16, 95\% CI 2.58-32.45). There was no statistically significant increase in the odds of mortality with typical comorbid conditions such as systemic sclerosis, Raynaud's syndrome, SLE, PBC, polymyalgia rheumatica, MGUS, RA, or history of bone marrow transplant.

Table II. Predictors of early unplanned readmissions for GAVE

\begin{tabular}{lcc}
\hline Characteristics & OR $(95 \% \mathrm{CI})$ & $\mathrm{p}$ \\
\hline Females & $1.03(0.93-1.14)$ & 0.539 \\
Age & $0.98(0.98-0.99)$ & 0.0001 \\
Portal hypertension & $1.64(1.37-1.95)$ & 0.0001 \\
Systemic sclerosis & $0.61(0.27-1.35)$ & 0.234 \\
Raynaud's syndrome & $0.76(0.29-1.98)$ & 0.57 \\
Systemic lupus erythematous & $0.93(0.45-1.91)$ & 0.84 \\
Primary biliary cholangitis & $1.60(0.69-3.69)$ & 0.26 \\
History of bone marrow transplant & $0.49(0.06-3.92)$ & 0.50 \\
Coronary artery disease & $0.97(0.86-1.11)$ & 0.72 \\
Polymyalgia rheumatica & $1.34(0.65-2.75)$ & 0.42 \\
Monoclonal gammopathy of & $1.15(0.51-2.61)$ & 0.72 \\
undetermined significance & & \\
Chronic kidney disease & $1.44(1.23-1.69)$ & 0.0001 \\
Rheumatoid arthritis & $0.84(0.57-1.24)$ & 0.399 \\
Non-alcoholic fatty liver disease & $1.42(0.88-2.27)$ & 0.142 \\
Cirrhosis & $1.57(0.62-3.93)$ & 0.335 \\
$\quad \begin{array}{l}\text { Alcoholic Cirrhosis } \\
\text { Autoimmune cirrhosis }\end{array}$ & Reference & Reference \\
\hline Common comorbid condions & & \\
\hline
\end{tabular}

Common comorbid conditions of GAVE and odds ratio of 30-day unplanned readmission

Table III. Multivariate regression analysis of mortality during index admission

\begin{tabular}{|c|c|c|}
\hline Characteristics & OR $(95 \% \mathrm{CI})$ & $\mathrm{p}$ \\
\hline Females & $1.0(0.62-1.59)$ & 0.99 \\
\hline Age & $1.005(0.98-1.02)$ & 0.59 \\
\hline Portal hypertension & $1.49(0.84-2.63)$ & 0.17 \\
\hline Systemic sclerosis & $1.5(0.31-7.16)$ & 0.61 \\
\hline Raynaud's syndrome & $1.42(0.28-7.05)$ & 0.66 \\
\hline Systemic lupus erythematous & $2.80(0.6711 .74)$ & 0.15 \\
\hline Primary biliary cholangitis & 1.00 & -- \\
\hline History of bone marrow transplant & 1.00 & -- \\
\hline Coronary artery disease & $0.79(0.52-1.22)$ & 0.303 \\
\hline Polymyalgia rheumatica & $3.74(0.47-29.29)$ & 0.20 \\
\hline $\begin{array}{l}\text { Monoclonal gammopathy of } \\
\text { undetermined significance }\end{array}$ & 1.00 & -- \\
\hline Chronic kidney disease & $0.83(0.49-1.38)$ & 0.47 \\
\hline Rheumatoid arthritis & $0.70(0.15-3.12)$ & 0.64 \\
\hline Nonalcoholic fatty liver disease & 1.00 & -- \\
\hline Cirrhosis & Reference & Reference \\
\hline Alcoholic Cirrhosis & 1.00 & -- \\
\hline Autoimmune cirrhosis & $9.16(2.58-32.45)$ & 0.001 \\
\hline
\end{tabular}

Multivariate regression analysis of factors associated with mortality in index admissions for GAVE-related bleeding

\section{DISCUSSION}

GAVE was first described by Rider et. al in 1953 in a patient with severe iron deficiency anemia [29]. While this is a relatively uncommon condition, GAVE-related gastrointestinal bleeding accounts for up to $4 \%$ of all non-variceal UGIB [11]. Typical clinical presentations can range from occult gastrointestinal bleeding and iron deficiency anemia to severe acute UGIB. Common comorbidities associated with GAVE are cirrhosis, vascular disease, $\mathrm{CKD}$, and autoimmune disease $[14,30]$. Treatment is challenging and options include surgical therapy, medical therapy, and most commonly, endoscopic therapy $[3,26]$.

Our study is the largest retrospective cohort study of GAVE utilizing the $2016 \mathrm{NRD}$. It showed that the 30 -day readmission rates for patients admitted for GAVE was relatively high at $20.49 \%$ and the 30 -day mortality of GAVE-related admissions was relatively low at $1.82 \%$. This mortality rate is similar to a previously described inpatient mortality rate of $1.4 \%$ [31]. Despite a relatively low 30 -day morality rate, the high rate of readmissions led to longer hospital stays and higher hospital costs, which has important individual patient and societal implications. Thus, strategies to prevent readmissions and rebleeding events in GAVE patients are needed to decrease healthcare costs and hospital length of stay.

Autoimmune hepatitis was noticed to have significantly higher odds of mortality with GAVE related bleeding during the index admission despite adjusting for confounders. There may be several explanations for this. It is possible that antibodies such as antinuclear antibodies (ANAs), anti-centromere and anti-RNA helicase II could have a cross-reaction with specific proteins in the gastric mucosa leading to GAVE [32]. Thus, it is possible that these patients may have ongoing inflammation due to autoimmune hepatitis which may lead to worsening of GAVE. Given the lack of laboratory markers in the NRD, it is not possible to determine if these patients were in remission or had an active disease. This could be a topic for further study to evaluate the impact of autoimmune hepatitis on outcomes of GAVE. Additionally, the NRD is unable to provide information if hemostasis was achieved after endoscopy. This would be an important clinical outcome that would provide valuable information for mortality. Therefore, further studies are required for the confirmation of our results.

Endoscopy during the index admission did not decrease the odds of readmission. This may be because GAVE is difficult to manage and tends to recur, even after endoscopic treatment. Multiple studies have demonstrated high recurrence rates of GAVE after APC treatment [33-35]. Additionally, multiple sessions of APC tend to be required to prevent rebleeding, but despite this, many patients still relapse [34]. Radiofrequency ablation seems to be a more promising treatment but studies are limited and mainly focus on patients previously refractory to APC treatment [36-38]. This analysis was unable to determine what treatment patients received, as there are no specific ICD$10 \mathrm{CM}$ codes for RFA, APC, or other endoscopic treatment. Comparison of readmission and rebleeding among endoscopic treatments should be a topic of future study.

Despite higher prevalence of comorbid conditions such as SLE in the readmitted group and Sjogren's, Raynaud's, and 
polymyalgia rheumatica, these conditions did not significantly increase the risk of early readmission in patients with GAVE. However, those with CKD and portal hypertension did have a statistically significant increased risk of early readmission. Similarly, younger patients had a slightly lower odds of readmission. While the readmitted patients were slightly younger, the statistics do not indicate important clinical significance. The OR for the age are significantly close to 1 and indicate a small, likely not clinically, significant decrease in the chances of readmission. Further studies are needed to confirm these findings.

Previous studies have not demonstrated that portal hypertension plays a role in the natural history of GAVE. Portal hypertension is not present in up to $70 \%$ of patients with GAVE. In patients with concomitant portal hypertension, treatment of portal hypertension with surgical portacaval shunts or transjugular intrahepatic portosystemic shunts does not appear to improve the course of GAVE $[39,40]$. In contrast to these reports, two clinical cases demonstrated the complete resolution of GAVE after liver transplantation in patients with persistent portal hypertension [41]. This suggests that the pathophysiology associated with liver failure, rather than portal hypertension, is responsible for the development of GAVE and correction of the liver failure alone is sufficient to cure GAVE.

It remains unclear as to why patients with portal hypertension had a higher risk of readmission. Subgroup analysis did not reveal any specific etiology of portal hypertension leading to increased readmission including NAFLD, autoimmune hepatitis, and alcoholic cirrhosis. While it does not appear that treatment of portal hypertension improves outcomes in GAVE, our data suggest that patients with portal hypertension are at higher risk of early readmission due to rebleeding. Thus, it may be reasonable to perform screening endoscopies on patients with known portal hypertension to identify and treat GAVE. Those with frequent rebleeding from GAVE should be screened for the presence of portal hypertension. Further research is needed to determine if specific interventions, such as APC or RFA, reduce the risk of early readmission and rebleeding.

The association of CKD and end-stage renal disease with GAVE is not well defined in the literature and is mainly described in case reports of patients with CKD, including those on hemodialysis [18, 42-45]. The pathogenesis of CKDassociated GAVE is unknown but has been theorized to be linked to poor renal excretion of vasoactive mediators such as gastric and prostaglandin E2, poor gastric motility, and uremic platelet dysfunction [18,46-48]. Identification of GAVE is critical in these patients as many patients with CKD have comorbid iron-deficiency anemia or erythropoietin-deficient anemia. Thus, further bleeding, even occult, may quickly cause severe anemia [49].

Strengths of our study include the large sample size and the capture of all readmissions utilizing a national readmission database in the United States over a 1-year period. This study includes patients admitted to community and academic medical centers across the United States thereby limiting selection bias. Given the use of ICD-10 CM codes to identify patients with GAVE related admissions and associated comorbidities, it is possible that patients with inaccurate coding may have been missed. Another potential weakness is the lack of information regarding the treatment modalities patients received. It would be interesting to see if specific treatments reduce readmissions among patients with GAVE, especially those with a high risk of early readmission. Another limitation would be that readmissions might be underestimated. This is possible as the NRD does not capture any follow up information for patients who underwent a clinic visit or for patients who died at home, en-route to the hospital, or in the emergency department. Despite these limitations, NRD, the largest national database, captures all readmissions after the index admission and provides valuable 30-day readmission information. Although clinic follow up for the index cohort after discharge is useful, 30-day readmission is the metric used for hospital reimbursement. Therefore, this study looked at the 30-day readmission rate and not clinical follow up, and specific studies for clinical follow up would be needed.

Our study is a large, comprehensive representation of 30-day readmission, 30-day mortality, demographic characteristics, and comorbidities for patients admitted for complications of GAVE. Further studies are required to determine if specific interventions may reduce the risk of readmission of GAVE patients, especially those with CKD and portal hypertension, who are at a high risk of early readmission. Investigation of screening for patients with recurrent bleeding from GAVE for CKD and portal hypertension should also be considered.

\section{CONCLUSIONS}

Our study demonstrates that the overall 30-day mortality rate of GAVE-related admissions is relatively low (1.82\%), but the 30-day readmission rate is significantly high (20.5\%). Patients with comorbid CKD and portal hypertension have a significantly higher risk of readmission.

\section{Conflicts of interest: None to declare.}

Authors contribution: L.P.: study design, data analysis, manuscript draft. A.B.: study design, data acquisition, statistical analysis, manuscript draft. H.M.: statistical analysis. P.R., D.R., and A.T.: study design, data collection and manuscript revision. A.P. and V.R.: critical revision of manuscript, study supervision. All authors agree to be accountable for all aspects of the work.

Supplementary material: To access the supplementary material visit the online version of the $J$ Gastrointestin Liver Dis at http://dx.doi. org/10.15403/jgld-804

\section{REFERENCES}

1. Jabbari M, Cherry R, Lough JO, Daly DS, Kinnear DG, Goresky CA. Gastric antral vascular ectasia: the watermelon stomach. Gastroenterology 1984;87:1165-1170. doi:10.1016/S00165085(84)80080-3

2. Thomas A, Koch D, Marsteller W, Lewin D, Reuben A. An Analysis of the Clinical, Laboratory, and Histological Features of Striped, Punctate, and Nodular Gastric Antral Vascular Ectasia. Dig Dis Sci 2018;63:966-973. doi:10.1007/s10620-018-4965-Z 
3. Zepeda-Gómez S, Sultanian R, Teshima C, Sandha G, Van Zanten S, Montano-Loza AJ. Gastric antral vascular ectasia: a prospective study of treatment with endoscopic band ligation. Endoscopy 2015;47:538-540. doi:10.1055/s-0034-1391395

4. Ripoll C, Garcia-Tsao G. The management of portal hypertensive gastropathy and gastric antral vascular ectasia. Dig Liver Dis 2011;43:345-351. doi:10.1016/j.dld.2010.10.006

5. Payen JL, Calès P, Voigt JJ, et al. Severe portal hypertensive gastropathy and antral vascular ectasia are distinct entities in patients with cirrhosis. Gastroenterology 1995;108:138-144. doi:10.1016/0016-5085(95)900187

6. Burak KW, Lee SS, Beck PL. Portal hypertensive gastropathy and gastric antral vascular ectasia (GAVE) syndrome. Gut 2001;49:866-872. doi:10.1136/gut.49.6.866

7. Gilliam JH 3rd, Geisinger KR, Wu WC, Weidner N, Richter JE. Endoscopic biopsy is diagnostic in gastric antral vascular ectasia. The “watermelon stomach”. Dig Dis Sci 1989;34:885-888. doi:10.1007/ bf01540274

8. Suit PF, Petras RE, Bauer TW, Petrini JL. Gastric antral vascular ectasia A histologic and morphometric study of "the watermelon stomach". Am J Surg Pathol 1987;11:750-757.

9. Vesoulis Z, Naik N, Maseelall P. Histopathologic changes are not specific for diagnosis of gastric antral vascular ectasia (GAVE) syndrome: a review of the pathogenesis and a comparative image analysis morphometric study of GAVE syndrome and gastric hyperplastic polyps. Am J Clin Pathol 1998;109:558-564. doi:10.1093/ajcp/109.5.558

10. Marsteller WF, Lewin DN, Reuben A. The biopsy GAVE the diagnosis. Clin Gastroenterol Hepatol 2012;10:e75-e76. doi: 10.1016/j. cgh.2011.12.024

11. Dulai GS, Jensen DM, Kovacs TO, Gralnek IM, Jutabha R. Endoscopic treatment outcomes in watermelon stomach patients with and without portal hypertension. Endoscopy 2004;36:68-72. doi:10.1055/s-2004-814112

12. Gretz JE, Achem SR. The watermelon stomach: clinical presentation, diagnosis, and treatment. Am J Gastroenterol 1998;93:890-895.

13. Maida M, Camilleri S, Manganaro M, Garufi S, Scarpulla G Radiofrequency Ablation for Treatment of Refractory Gastric Antral Vascular Ectasia: A Systematic Review of the Literature. Gastroenterol Res Pract 2017;2017:5609647. doi:10.1155/2017/5609647

14. Gostout CJ, Viggiano TR, Ahlquist DA, Wang KK, Larson MV, Balm R. The clinical and endoscopic spectrum of the watermelon stomach. J Clin Gastroenterol 1992;15:256-263. doi:10.1097/00004836-199210000-00019

15. Hung EW, Mayes MD, Sharif R, et al. Gastric antral vascular ectasia and its clinical correlates in patients with early diffuse systemic sclerosis in the SCOT trial. J Rheumatol 2013;40:455-460. doi:10.3899/ jrheum. 121087

16. Marie I, Levesque H, Ducrotté $P$, et al. Gastric involvement in systemic sclerosis: a prospective study. Am J Gastroenterol 2001;96:77-83.

17. Tobin RW, Hackman RC, Kimmey MB, et al. Bleeding from gastric antral vascular ectasia in marrow transplant patients. Gastrointest Endosc 1996;44:223-229. doi:10.1016/s0016-5107(96)70155-4

18. Stefanidis I, Liakopoulos V, Kapsoritakis AN, et al. Gastric antra vascular ectasia (watermelon stomach) in patients with ESRD. Am J Kidney Dis 2006;47:e77-e82. doi:10.1053/j.ajkd.2006.02.185

19. Ito K, Shiraki K, Sakai T, Yoshimura H, Nakano T. Portal hypertensive colopathy in patients with liver cirrhosis. World J Gastroenterol 2005;11:3127-3130. doi:10.3748/wjg.v11.i20.3127

20. Menchén L, Ripoll C, Marín-Jiménez I, et al. Prevalence of portal hypertensive duodenopathy in cirrhosis: clinical and haemodynamic features. Eur J Gastroenterol Hepatol 2006;18:649-653. doi:10.1097/00042737-200606000-00012

21. Bresci G, Parisi G, Capria A. Clinical relevance of colonic lesions in cirrhotic patients with portal hypertension. Endoscopy 2006;38:830835. doi:10.1055/s-2006-944629

22. Figueiredo P, Almeida N, Lérias C, et al. Effect of portal hypertension in the small bowel: an endoscopic approach. Dig Dis Sci 2008;53:21442150. doi:10.1007/s10620-007-0111-Z

23. Higaki N, Matsui $H$, Imaoka $H$, et al. Characteristic endoscopic features of portal hypertensive enteropathy. J Gastroenterol 2008;43:327-331. doi:10.1007/s00535-008-2166-9

24. Primignani M, Carpinelli L, Preatoni P, et al. Natural history of portal hypertensive gastropathy in patients with liver cirrhosis. The New Italian Endoscopic Club for the study and treatment of esophageal varices (NIEC). Gastroenterology 2000;119:181-187. doi:10.1053/ gast. 2000.8555

25. Merli M, Nicolini G, Angeloni S, Gentili F, Attili AF, Riggio O. The natural history of portal hypertensive gastropathy in patients with liver cirrhosis and mild portal hypertension. Am J Gastroenterol 2004;99:1959-1965.

26. Tran A, Villeneuve JP, Bilodeau M, et al. Treatment of chronic bleeding from gastric antral vascular ectasia (GAVE) with estrogen-progesterone in cirrhotic patients: an open pilot study. Am J Gastroenterol 1999;94:2909-2911

27. NRD Overview [Internet]. Overview of the Nationwide Readmissions Database (NRD). [Cited 2020 Jan 2]. Available from: https://www. hcup-us.ahrq.gov/nrdoverview.jsp

28. HCUP-US SID Overview [Internet]. Overview of the State Inpatient Databases (SID). [Cited 2020 Jan 2]. Available from: https://www.hcupus.ahrq.gov/sidoverview.jsp

29. Rider JA, Klotz AP, Kirsner JB. Gastritis with veno-capillary ectasia as a source of massive gastric hemorrhage. Gastroenterology 1953;24:118123. doi:10.1016/S0016-5085(53)80070-3

30. Ward EM, Raimondo M, Rosser BG, Wallace MB, Dickson RD. Prevalence and natural history of gastric antral vascular ectasia in patients undergoing orthotopic liver transplantation. J Clin Gastroenterol 2004;38:898-900. doi:10.1097/00004836-200411000-00013

31. Patel U, Desai R, Desai J, et al. Predictors of blood transfusion and in-hospital outcomes in patients with gastric antral vascular ectasia (GAVE): a nationwide population-based analysis. Ann Transl Med 2019;7:46. doi:10.21037/atm.2019.01.11

32. Fuccio L, Mussetto A, Laterza L, Eusebi LH, Bazzoli F. Diagnosis and management of gastric antral vascular ectasia. World J Gastrointest Endosc 2013;5:6-13. doi:10.4253/wjge.v5.i1.6

33. Boltin D, Gingold-Belfer R, Lichtenstein L, Levi Z, Niv Y. Long-term treatment outcome of patients with gastric vascular ectasia treated with argon plasma coagulation. Eur J Gastroenterol Hepatol 2014;26:588593. doi:10.1097/MEG.0000000000000047

34. Fuccio L, Zagari RM, Serrani M, et al. Endoscopic argon plasma coagulation for the treatment of gastric antral vascular ectasia-related bleeding in patients with liver cirrhosis. Digestion 2009;79:143-150. doi:10.1159/000210087

35. Chaves DM, Sakai P, Oliveira CV, Cheng S, Ishioka S. Watermelon stomach: clinical aspects and treatment with argon plasma coagulation. Arq Gastroenterol 2006;43:191-195. doi: 10.1590/s000428032006000300007

36. Markos P, Bilic B, Ivekovic H, Rustemovic N. Radiofrequency ablation for gastric antral vascular ectasia and radiation proctitis. Indian J Gastroenterol 2017;36(2):145-8. 
37. Dray X, Repici A, Gonzalez P, Fristrup C, Lecleire S, Kantsevoy S, et al. Radiofrequency ablation for the treatment of gastric antral vascular ectasia. Endoscopy 2014;46(11):963-9.

38 McGorisk T, Krishnan K, Keefer L, Komanduri S. Radiofrequency ablation for refractory gastric antral vascular ectasia (with video). Gastrointest Endosc 2013;78:584-588. doi:10.1016/j. gie.2013.04.173

39. Spahr L, Villeneuve JP, Dufresne MP, et al. Gastric antral vascular ectasia in cirrhotic patients: absence of relation with portal hypertension. Gut 1999;44:739-742. doi:10.1136/gut.44.5.739

40 Kamath PS, Lacerda M, Ahlquist DA, McKusick MA, Andrews JC, Nagorney DA. Gastric mucosal responses to intrahepatic portosystemic shunting in patients with cirrhosis. Gastroenterology 2000;118:905-911. doi:10.1016/s0016-5085(00)70176-4

41 Vincent C, Pomier-Layrargues G, Dagenais M, et al. Cure of gastric antral vascular ectasia by liver transplantation despite persistent portal hypertension: a clue for pathogenesis. Liver Transpl 2002;8:717-720. doi:10.1053/jlts.2002.34382

42. Rimševičius L, Galkauskas D, Lavinskas J, et al. Gastric antral vascular ectasia should not be overlooked in erythropoietin resistance: a series of case reports. Acta Med Litu 2018;25:219-225. doi:10.6001/actamedica. v25i4.3932
43. Iguchi A, James Kazama J, Komatsu M, et al. Three Cases of Gastric Antral Vascular Ectasia in Chronic Renal Failure. Case Rep Nephrol Urol 2011;1:15-19. doi:10.1159/000332832

44. Pisharam JK, Ramaswami A, Chong VH, Tan J. Watermelon stomach: a rare cause of anemia in patients with end-stage renal disease. Clin Nephrol 2014;81:58-62. doi:10.5414/CN107527

45. Lata S, Gupta V, Nandwani A, Sharma P. Watermelon stomach in end-stage renal disease patient. Indian J Nephrol 2012;22:477-479. doi:10.4103/0971-4065.106055

46. Quintero E, Pique JM, Bombi JA, et al. Gastric mucosal vascular ectasias causing bleeding in cirrhosis. A distinct entity associated with hypergastrinemia and low serum levels of pepsinogen I. Gastroenterology 1987;93:1054-1061. doi:10.1016/0016-5085(87)90569-5

47. Lowes JR, Rode J. Neuroendocrine cell proliferations in gastric antral vascular ectasia. Gastroenterology 1989;97:207-212. doi:10.1016/00165085(89)91437-6

48. Saperas E, Perez Ayuso RM, Poca E, Bordas JM, Gaya J, Pique JM. Increased gastric PGE2 biosynthesis in cirrhotic patients with gastric vascular ectasia. Am J Gastroenterol 1990;85:138-144.

49. Lee DJ, Fragata J, Pestana JO, et al. Erythropoietin resistance in endstage renal disease patient with gastric antral vascular ectasia. J Bras Nefrol 2015;37:410-413. doi:10.5935/0101-2800.20150062 